of the same offensive chraracter, but much slighter in degree to that before mentioned.

Thus far all that has been shown is that in the organs of the body after deatb, and from the air, \&c., certain microbes have been obtained, and that these have been artificially cultivated in nutrient media; but no direct evidence is forthcoming to show their connexion with the disease. I am, however, able to assert from actual experiment that a very small dose of these artificially cultivated microbes will produce a smart attack of diarrhoea; and although it is far from being proved that this diarrhoea is the result of some form of bacterial life, as the four essential conditions for any such proof are not yet forthcoming-i.e. (1) the presence of a distinct and specific organism in the blood or tissues of those suffering from the disease, (2) the artificial cultivation of these apart from the body, (3) the production of the disease by introducing these into a healthy body, \&c., and (4) the presence of the same organism again in the body when such disease is introduced,-yet I would submit that one is justified in saying that there is evidence to connect these organisms with the prevalence of the disease; and although this slight contribution to a most obscure and difficult subject is little more than preliminary to the complete study of the etiology of summer diarrhoea, and is open to eriticism at every point, I have thought it not out of place to bring together, as it were, to a focus what little in the intervals of other work I have had the opportunity of doing. Town Hall, Loicester.

\section{A CASE OF HYDROPHOBIA AND A CASE OF TETANUS.}

BY H. HANDFORD, M.D., M.R.C.P., pHYiclaN TO THE NOT'TNGHAM GENERAL HOSPTTAL.

W. P-, a powerfully built carter, aged thirty-two, was admitted under my care into the Nottingham General Hospital, at 8 A.M., on Feb. 3rd, 1887, said to be suffering from hydrophobia. He had been bitten by a stray dog four months and a quarter previously. No further information could be gained about the dog except that it was not known in the neighbourhood in which the patient lived. The wounds were on the back of the third and fourth fingers of the right hand; they were not cauterised. He treated them with domestic remedies, and they healed in a week or ten days. The scars were quite evident, and had not become painful or irritable. When I saw him a few hours after his admission and examined him, though I led him up to the subject several times, he markedly avoided any mention of his having been bitten, or of his difficulty in swallowing, until directly asked. There was a very evident unwillingness to talk or even think of the subject. This at the time seemed to me to point strongly in favour of the genuine nature of the disease, for at the outset there were many difficulties in the diagnosis of hydrophobia, which had been first made by the patient himself before coming to the hospital. He had been a soldier, had had "fever" in India, and had been very intemperate. His mother died insane, and a brother has "fits." There was some doubt whether the patient had been drinking for the previous fortnight, during which time his wife said he did not segm in his usual health, but be did not speak of the bite. The patient himself said that three days before his admission he began to feel ill and thought he had a "cold." He was sleepless, restless, dizzy, and had momentary losses of vision. He consulted a medical man, who prescribed for him. After he returned home, as he was washing, he screamed directly the water touched his face. Shortly afterwards he noticed some difficulty in swallowing. He then made the diagnosis of hydrophobia for himeelf, went again to his doctor (whom he had not previously told either of the bite or of his difficulty in swallowing), and he confirmed it. A few hours later he walked to the hospital and asked to be admitted. He complained of diffculty of swallowing, and of a feeling of constriction over the upper part of the sternum. His expression was very anxious, eyes rather wild and staring, and he spoke with an appearance of enforced calmness. The temperature was normal; pulse 56, and quite regular; respiration 22 , and markedly irregular, being frequently interrupted by deep sighs. My attention was directed to the character of the respiration by my colleague, MIr. White, whose experience extends to six or seven cases, in all of which irregularity of respiration was very noticeable. There was no huskiness or alteration of the voice, and no frothing at the mouth or spitting till a few minutes before death. His tongue was fissured, soft, flabby, and coated in patches with a thin white fur. He complained of loss of appetite and thirst, and had taken very little food for three days. He vomited several times the day before his admission. His urine was rather concentrated, sp. gr. 1024, and contained no albumen. At 10 A.M., two hours after his admission, the case was taken by Dr. Williams, and much of the following is taken from his notes. The patient was quite rational and could give a clear account of his illness. He asked for something to drink almost as soon as he came in. He would not let the nurse hold the cup, but grasped it himself quietly and firmly and swallowed the water at one gulp. During this act he became much excited. He would not have more than a tablespoonful of water in the cup at a time. He said he had no difficulty in swallowing, all his difficulty being in getting the cup to his mouth. When he was about to be washed, as is usual, he became much excited, and said to the sister of the ward, "Don't bring water near me." He asked to be sponged instead, but said if he had to be washed he would do his best not to scream. The noise of pouring out water at this time did not affect him. When washed he shuddered and sighed like a person in a cold bath, and turned his head away from the water, but was otherwise unaffected till his face was to be washed, when he asked for the sponge and himself rubbed his face violently. In doing so he squeezed out a few drops of water, which trickled down his face and caused a slight convulsion. When I saw him at mid-day he was quite calm and rational. On my requesting to see him drink, he asked me to stand away, turned his head from the locker on which the cup stood, reached out his hand, seized the cup, and brought it rapidly to his mouth with very irregular, jerking movements, took a large mouthful and swallowed it with marked effort, accompanied by very slight facial spasm and some general excitement. The process of swallowing was much like that of a child taking disagreeable medicine. There was nothing that one could fairly call a convulsion at this time, but there was probably incoördination of the muscles of deglutition. At 8 P.M. I saw him again, and he said he felt much better. His temperature remained normal, his expression was less anxious, pupils widely dilated, pulse full, quiet, and regular. During the day he had taken about a teacupful of rice pudding without much difficulty, and had drunk about a pint and a half of milk. He was so much improved at this time that a medical friend who saw him with me, and who had previously seen several cases of hydrophobia, entertained much doubt as to the genuineness of the disease. The patient was ordered an enema of chloral and bromide of potassium. It was given at 8.30 P.M. without difficulty, and he went to sleep between 11 and 12 P.M. After some broken sleep he finally woke at 5 A.M. on Feb. 4th, and suddenly manifested marked delusions, and became furiously maniacal. He had illusions of sight, and wanted to go through the window. He was a very powerful man, and it was with great difficulty that he could be restrained in bed. There were intervals of comparative calm, during which he could be reasoned with, but without effect. He expressed a wish not to hurt anyone, but during his furious delirium made determined efforts to bite the hands of those restraining him, The excitement was greater than anything I have witnessed in delirium tremens or in mania. He was treated by the hypodermic injection of morphia. At this time the noise of pouring out water gave him a convulsion. He was perspiring profusely, and the act of wiping the face with a handkerchief caused a spasm, as also did the draught of air caused by moving a towel near him. He said he was hungry, and called for meat. Some bread-and-butter was given him. He masticated it, but could not swallow it. He took water from a spoon, but refused a cup; the act of swallowing caused great excitement, and also spasms of the muscles of deglutition, lasting on the whole nearly a minute. In the intervals, though appearing rational, his statements were incorrect. For instance, he said he had been bitten only five weeks ago, and on the knee. The day before he had given a correct account. Talking of the dog or of the bite agitated him greatly. Among other illusions he saw a dog under the bed, He made most heart-rending appeals to those restraining him in bed, and taunted them with gloating over his misery. His death took place somewhat suddenly, apparently from 
failure of the heart, twenty-eight hours after his admission into the hospital, and on the third day (little more than forty-eight hours) after difficulty of swallowing was first noticed. Half an hour before death he could swallow milk, but sputtered out some of it. From this time he was frothing at the mouth, hawking up and spitting saliva. Twenty minutes after death rigor mortis had commenced, and the temperature in the rectum was $1088^{\circ}$. There had been but little spasm of the trunk or limbs during life, though much voluntary tffort. No priapism or sexual excitement was detected.

The body was examined three hours after death. Rigor mortis was strongly marked, and post-mortem lividity of the dependent parts of the body was distinct. The spinal cord was removed first. The blood was dark and remarkably fluid. Nearly two pints of blood escaped during the removal of the cord. The brain was next removed. There was a moderate amount of subarachncid effusion and congestion of the small vessels in the pia mater. The cortical grey matter of the brain and the adjacent white substance for about one-third of an inch had an evident pink flush, due to capillary congestion. There were no hæmorrhages visible to the naked eye. The larynx was examined, and the interior (especially the false cords) was dusky and much congested. The heart was dilated, but empty. The other organs appeared healthy. Parts of the medulla and of the cervical cord were at once placed in powdered boric acid and forwarded to the Brown Institute, and I hear, through the kindness of Mr. Victor Horsley, that rabbits inoculated with them died of typical rabies. Other parts were carefully hardened in bichromate of ammonia solution, but sections showed nothing beyond an unusual fulness of the vessels, many of which were thrombosed, but this was probably a post-mortem effect. No cellular infiltration or unusual number of leucocytes could be found, either in the medulla near the origin of the vagus nor in the cervical cord, though carefully looked for. In two cases of rabies in the dog sections of the medulla and cord were examined, but nothing beyond marked fulness and dilatation of the ressels could be found. There was no cellular infiltration or distinct evidence of an inflammatory condition. The disease is apparently too little localised and too rapid in its progress for coarse tissue changes to be found.

In this case difficulty of swallowing was not a particularly prominent symptom, neither would changes in the medulla account for the furious maniacal excitement which with its sudden onset were the marked features of the case. During the early stage the symptoms were so slight, and their progress so little, that many considered the case one of nervous mimicry; while those who only saw him during the stage of delirium, taking into account the family history of insanity and the drinking habits of the patient, looked upon it as acute mania. The complete clinical picture, however, leaves no doubt, even if the inoculation experiments had not been performed. To sum up the special features of the case, the incubation period was long-four months and a quarter,- the early symptoms very slight and slow in progress, the outburst of delirium very sudden and its intensity unusually great, and the stage of excitement very shortseven hours.

As a contrast to the above, I may give a few details of the following case of tetanus which came under my care a few weeks later. It was at first supposed to be idiopathic tetanus - a disease of very doubtful existence-but a careful search resulted in the discovery of a wound. The history is as follows:-

On Monday, April 4th, J. W-, a labourer, aged twenty-eight years, noticed a sore on the sole of his foot, caused by a nail in his boot; the place had inflamed and "gathered," and was very painful. (On admission into the hospital this sore was completely healed, and not in any way painful.) On Tuesday he rested from work. On Wednesday he worked in the market all day. On Thursday morning he complained of his jaws feeling stiff. On Friday morning he walked a distance of two miles, and on reaching home burst into tears, saying he had fallen down four times. His jaws were now much more firmly closed, and he complained of great pain and a feeling of constriction round the lower part of the thorax, and of dizziness. He went to bed, and shortly after had an attack of opisthotonos. On Saturday his jaws were rery firmly closed, and he was given twenty grains of chloral every two hours. In the evening the dose was increased to thirty grains. On Sunday he was much better. On Monday he was admitted into the hospital. $\mathrm{He}$ was very drowsy from the chloral, but when roused gave a clear account of his illness. He could open the mouth well, and during the day took three pints of milk without difficulty. The tonic spasm of the muscles closing the jaws had almost entirely passed off, neither was there any permanent arching of the back (from tonic spasm of the erector spinæ) or hardness of the abdomen. Possibly this was the effect of the large doses of chloral, which, however, had no effect upon the clonic spasms, which came on, without any apparent irritation from noise or draught, every two or three minutes. The spasm of the diaphragm, as evidenced by a sharp inspiration and pain at the epigastrium, was marked during each convulsion. The ease with which fluids could be taken without bringing on any spasm was remarkable. The patient was so drowsy on admission that the chloral was not resumed till evening, when he was ordered thirty grains every four hours, and he had the last dose at 5.45 A.M. on Tuesday. During the night he took only half a pint of beef-tea, as the difficulty of swallowing had increased. 0n Tuesday morning, at 6 A M., it was not thought safe to give him any more chloral, as he was very drowsy; face inclining to be dusky; breathing noisy, 43 per minute; pulse 134 per minute; pupils contracted, but reacting to light. The general clonic spasms, which the previous evening had been from twenty to twenty-four in the hour, were still sixteen or eighteen-that is, coming on rather more frequently than once in four minutes. During the day he received nutrient and stimulant enemata, but the spasms continued at the rate of from four to fifteen in the hour, and he died at 7 P.M., the pulse failing some time before the respiration. Death took place on the sixth day after the commencement of the symptoms. An inspection of the body was made twelve hours afterwards. There was no undue fluidity of the blood, and no naked-eye change in any of the viscera. Portions of the spinal cord and of the posterior tibial nerve on the affected side were prepared for microscopic examination.

The points of interest are: the temporary improvement under the use of chloral; the cessation, to a very large extent, of the tonic spasm, and the facility in swallowing the failure of the chloral, though pushed as far as was afe, to materially influence the general clonic convulsions, which remained severe till the last few hours of life; death from failure of the heart, rather than from spasm of the respiratory muscles during a convulsion, as is common.

\section{A CASE OF HYSTERICAL SPASM SIMU. LATING TETANY.}

BY F. FOORD CAIGER, M.D. LOND.

E. S-, a general servant, unmarried, was admitted into the Paddington Infirmary on Jan. 27th in a very prostrate condition. It was afterwards ascertained that she had always been delicate, but had had no serious illness until two years ago, when she was admitted into the Kensington Infirmary suffering from general weakness. Her teeth then became bad, and she had severe neuralgic pain in the face, and at the same time suffered from "cramps and fits." She stated that she then went out of her mind, and was removed to Leavesden Asylum, where she remained six weeks, and eventually went out to service. In her situation she was underfed, and at the same time was worked very hard. The catamenia have always been regular in time, but profuse. On the night of the 24th she was suddenly seized witli cramps, beginning in the left hand and foot, extending up the limbs, and affecting the left side of the trunk, which gave her great pain. At the same time the left side of the face became rigid. This, in its severity, passed off in about half an hour, but continued more or less until she was brought to the infirmary three days later.

On admission the following notes were taken: Pale woman; very prostrate; surface cold; respiration hurried and shallow; complaining of painful cramp in the left arm, leg, and side. The fingers of the left hand are flexed, and adducted at the proximal joints and extended at the distal. The thumb is rigidly flexed into the palm, which is bollowed by contraction of the hypothenar muscles. The hand is thus bent into the form of a rigid cone. The wrist is partially flexed, and the forearm semiflexed and pronated with some degree of rigid adduction of the shoulder joint. An analogous condition is present in the foot, the ankle 